\title{
Optimized Control of a Hydraulic Recuperation System for Skip Trucks
}

\author{
Robin Masser, Karsten Schwalbe, Karl Heinz Hoffmann \\ Technische Universität Chemnitz \\ Str. der Nationen 62, 09111 Chemnitz, Germany
}

robin.masser@physik.tu-chemnitz.de; karsten.schwalbe@physik.tu-chemnitz.de; hoffmann@physik.tu-chemnitz.de

\section{Extended Abstract}

Hydraulic hybrid powertrain architectures offer a great opportunity to reduce the energy consumption of vehicles by recovering brake energy and using it for propulsion, and have therefore been in the focus of research during the last decades.

Our work aims to minimize the fuel consumption of commercial vehicles, in particular skip trucks, with the use of a hydraulic recuperation system consisting of a bladder accumulator, a hydraulic pump/motor that is directly driven by the cardan shaft, a pressure control valve and a heat exchanger connecting the hydraulic cycle with the cooling cycle of the truck. This specific application does not only aim at the frequent acceleration and deceleration phases in urban areas but also enables supporting the thermal management of the vehicle and operating its skipping mechanism without the need for a running combustion engine.

Focusing a fuel-efficient use of the saved energy and optimal interaction of combustion engine and hydraulic pump/motor, we model the recuperation system applying Endoreversible Thermodynamics [1]. The hydraulic components of the system as well as efficiencies of both the combustion engine and the hydraulic pump/motor are incorporated in the model. In this way, the efficiency-optimized operation of both engines can be examined in mutual influence.

Based on the developed model, a section of urban driving data recorded from a real skip truck is used for optimization. The objective function of the optimization is the overall fuel consumption in the considered time interval. We examine the optimization with two different conditions: First, while the power output of the internal combustion engine and the hydraulic pump/motor add up to the desired power at the cardan shaft, the power output of the combustion engine can not exceed the desired power at the drive shaft. This corresponds to a support of the propulsion by the hydraulic pump/motor only using stored braking energy. And secondly, the power output of the combustion engine can exceed the desired power at the drive shaft, so that accelerating the truck and charging the bladder accumulator can be performed at the same time. For comparison, we also show a simple, non-optimized control where the propulsion is supported as soon as stored energy is available. The optimization itself is solved by time discretization of the model and non-linear programming for minimization.

In the case of the simple control strategy, the resulting fuel consumption of the skip truck is reduced by $22 \%$ in comparison to a skip truck without a recuperation system. By optimizing the control of the hydraulic unit according to the first mentioned conditions, fuel savings can be increased to $32 \%$. It turns out that the combustion engine is preferably operated in ranges of good efficiency alone or is almost completely replaced by the hydraulic unit. Using the second mentioned conditions leads to a further, albeit small, improvement of the fuel saving values to more than $35 \%$ while efficiency values of both combustion engine and hydraulic pump/motor increase. These results of our introduced parallel hydraulic hybrid setup demonstrate that fuel economy and emission performance can be improved significantly with a simple control strategy and even further by applying an optimized control strategy.

\section{References}

[1] K. H. Hoffmann, J. M. Burzler and S. Schubert, "Endoreversible Thermodynamics," J. Non-Equilib. Thermodyn., vol. 22, no. 4, pp. 311-355, 1997. 